\title{
A Clinical Survey Efficacy Of Frankincense In Acute Ischemic
} Stroke

\author{
Nahid Jivad ${ }^{1 *}$, Fakhri Rezaei kheirabadi ${ }^{2}$, M ahmoud Rafeian $^{3}$, Parisa Yarmohammadi ${ }^{4}$ \\ ${ }^{1}$ Associate professor, Department of Neurology, Faculty of M edicine, Shahrekord University of M edical Sciences, \\ Shahrekord, Iran. \\ ${ }^{2}$ Student of M edicine, Faculty of Medicine, Shahrekord University of Medical Sciences, Shahrekord, Iran. \\ ${ }^{3}$ Professor, Department of Pharmacology, Faculty of M edicine,Shahrekord University of M edical Sciences, \\ Shahrekord, Iran. \\ ${ }^{4} \mathrm{PhD}$ student, Department of animal physiology, University of Shiraz, Shiraz, Iran. \\ *Corresponding Author: Email: ఏivad@skums.ac.ir
}

Background \&Aims: Ischemic stroke is the most common cause of stroke, and paralysis and movement disorders are the most symptoms of stroke. Therefore, it is important to find a method for improving the disorders. This study aimed to examine the clinical efficacy of frankincense in patients with the acute ischemic stroke.

Methods: In this clinical trial, 60 patients with ischemic stroke were randomly allocated into the treatment and control groups $(n=30)$. In the treatment group, in addition to routine treatment of stroke, four capsules containing $500 \mathrm{mg}$ powdered frankincense was given daily , but in the control group, was performed only the treatment of stroke (no frankincense). The treatment lasted for 1 month. The NIHSS scale (for assessment of speech and muscle strength) at the beginning, the seventh day and end of the study for each patient was completed. Statistical analysis was performed using independent t-test, chi-square, mann withney in SPSS software.

Results: Results showed that only improvement of muscle strength in patients left limb increased significantly in stage II in the treatment group than the control group.

Conclusion: Adding frankincense to treatment of patients with stroke can be effective on improvement muscle strength in patients with muscle weakness and non-dominant side in acute neurological disorders. While that is not effective on improving muscle strength of the right limb or speech.

Key words: Stroke, Frankincense, M ovement disorder, Speech disorder

DOI: $10.7575 /$ aiac.abcmed.ca1.32

Published Date: February 2017

Peer-review is under responsibility of the 9th Iranian Stroke Congress.

Published by Australian International Academic Centre, Australia

This published work is open access under the CC BY license.

Available online at www.abcmed.aiac.org.au 